\title{
Process $e^{+} e^{-} \rightarrow 3 \pi(\gamma)$ with final state radiative corrections \\ S. Bakmaev * Yu. M. Bystritskiy it and E. A. Kuraet \\ Joint Institute for Nuclear Research, 141980 Dubna, Russia
}

(Dated: November 12, 2018)

\begin{abstract}
We consider the process of annihilation of $e^{+} e^{-}$to three pion final state for the case of moderately high energies. The final state emission of virtual and real photon is considered explicitly. The calculations are performed in frames of QED with pointlike mesons and pion part of chiral perturbation theory. Some numerical estimates are given.
\end{abstract}

\section{INTRODUCTION}

The problem of taking into account of radiative corrections ( $\mathrm{RC}$ ) of lowest order of perturbation theory to process of three pion production in annihilation channel of colliding $e^{+} e^{-}$at moderately high energies becomes urgent for precision measurement of hadronic contribution to muon anomalous magnetic moment $(g-2)_{\mu}$ [1]. This is the motivation of this paper. The similar calculation for production of $2 \pi$ and $\mu^{+} \mu^{-}$at the annihilation channel was performed recently [2].

We shall use the pion sector of chiral perturbation theory (ChPT) to perform the calculations of interaction of pions with electromagnetic field. This theory is given by WessZumino-Witten effective lagrangian [3, 4]. The relevant piece of this lagrangian is reproduced

*Electronic address: bakmaev@theor.jinr.ru

${ }^{\dagger}$ Electronic address: bystr@theor.jinr.ru

‡Electronic address: kuraev@theor.jinr.ru 
below:

$$
\begin{aligned}
& \mathcal{L}=\frac{f_{\pi}^{2}}{4} S p\left[D_{\mu} U\left(D^{\mu} U\right)^{+}+\chi U^{+}+U \chi^{+}\right]- \\
&- \frac{e}{16 \pi^{2}} \varepsilon^{\mu \nu \alpha \beta} A_{\mu} S p\left[Q\left\{\left(\partial_{\nu} U\right)\left(\partial_{\alpha} U^{+}\right)\left(\partial_{\beta} U\right) U^{+}-\left(\partial_{\nu} U^{+}\right)\left(\partial_{\alpha} U\right)\left(\partial_{\beta} U^{+}\right) U\right\}\right]- \\
&-\frac{i e^{2}}{8 \pi^{2}} \varepsilon^{\mu \nu \alpha \beta}\left(\partial_{\mu} A_{\nu}\right) A_{\alpha} S p\left[Q^{2}\left(\partial_{\beta} U\right) U^{+}+Q^{2} U^{+}\left(\partial_{\beta} U\right)+\right. \\
&\left.\quad+\frac{1}{2} Q U Q U^{+}\left(\partial_{\beta} U\right) U^{+}-\frac{1}{2} Q U^{+} Q U\left(\partial_{\beta} U^{+}\right) U\right],
\end{aligned}
$$

where $f_{\pi}=94 \mathrm{MeV}$ is the pion decay constant, $U=\exp \left(i \frac{\sqrt{2} \Phi}{f_{\pi}}\right), D_{\mu} U=\partial_{\mu} U+i e A_{\mu}[Q, U]$, $Q=\operatorname{diag}\left(\frac{2}{3},-\frac{1}{3},-\frac{1}{3}\right)$ is the quark charge matrix and terms with $\chi=\operatorname{Bdiag}\left(m_{u}, m_{d}, m_{s}\right)$ introduce explicit chiral symmetry breaking due to nonzero quark masses. The constant $\mathrm{B}$ has dimension of mass and is determined through the equation $B m_{q}=M^{2}$, where $m_{q}=$ $m_{u} \approx m_{d}$ and $M$ is the pion mass. The pseudoscalar meson matrix $\Phi$ has its standard form:

$$
\Phi=\left(\begin{array}{ccc}
\frac{1}{\sqrt{2}} \pi^{0}+\frac{1}{\sqrt{6}} \eta & \pi^{+} & K^{+} \\
\pi^{-} & -\frac{1}{\sqrt{2}} \pi^{0}+\frac{1}{\sqrt{6}} \eta & K^{0} \\
K^{-} & \bar{K}^{0} & -\frac{2}{\sqrt{6}} \eta
\end{array}\right) .
$$

\section{BORN APPROXIMATION}

We consider reaction of $e^{+} e^{-}$annihilation into three pions:

$$
e^{-}\left(p_{-}\right)+e^{+}\left(p_{+}\right) \rightarrow \pi^{-}\left(q_{-}\right)+\pi^{+}\left(q_{+}\right)+\pi^{0}\left(q_{0}\right)
$$

Matrix element for this process in Born approximation have a form (see Fig. 11):

$$
M^{(0)}=\frac{i \alpha}{\pi f_{\pi}^{(0) 3}} \cdot \frac{1}{q^{2}} \cdot \bar{v}\left(p_{+}\right) \gamma_{\mu} u\left(p_{-}\right) \cdot\left(\mu q_{+} q_{-} q_{0}\right)
$$

where $f_{\pi}^{(0)}$ is the unrenormalized pion decay constant, $s=q^{2}=\left(p_{+}+p_{-}\right)^{2}-$ invariant mass of initial state, $\left(\mu q_{+} q_{-} q_{0}\right) \equiv \varepsilon_{\mu \nu \alpha \beta} q_{+}^{\nu} q_{-}^{\alpha} q_{0}^{\beta}$.

Squaring this matrix element and performing summation over initial leptons spin states we get the total cross section for this process:

$$
\sigma_{B}^{(0)}=\frac{\alpha^{2} s^{2}}{2^{8} \cdot 3 \cdot \pi^{5} f_{\pi}^{(0) 6}} \int_{x_{+}^{\min }}^{x_{+}^{\max }} d x_{+} \int_{x_{-}^{\min }}^{x_{-}^{\max }} d x_{-} G\left(x_{+}, x_{-}\right)
$$

here

$$
G\left(x_{+}, x_{-}\right)=4\left(x_{+}^{2}-\mu^{2}\right)\left(x_{-}^{2}-\mu^{2}\right)-\left(1-2 x_{+}-2 x_{-}+2 x_{+} x_{-}+\mu^{2}\right)^{2},
$$


with $\mu^{2}=M^{2} / s, M$ - is pion mass, $x_{ \pm}=\varepsilon_{ \pm} / \sqrt{s}, x_{0}=\varepsilon_{0} / \sqrt{s}$ - are fractions of final pion's energies, while $x_{+}+x_{-}+x_{0}=1$. Limits of integration in (5) follows from kinematical constrains:

$$
\begin{aligned}
x_{+}^{\text {min }} & =\mu, \quad x_{+}^{\max }=\frac{1}{2}\left(1-\frac{3 M^{2}}{s}\right)=\frac{1}{2}\left(1-3 \mu^{2}\right), \\
x_{-}^{\text {max }, \text { min }} & =\frac{1}{2\left(1-2 x_{+}+\mu^{2}\right)}\left(\left(1-x_{+}\right)\left(1-2 x_{+}+\mu^{2}\right) \pm R\left(x_{+}\right)\right),
\end{aligned}
$$

where $R^{2}\left(x_{+}\right)=\left(x_{+}^{2}-\mu^{2}\right)\left(1-2 x_{+}+\mu^{2}\right)\left(1-2 x_{+}-3 \mu^{2}\right)$.

\section{VIRTUAL PHOTON EMISSION}

Radiative corrections from emission of virtual photon can be represented by 11 Feynman diagrams (FD) (see Fig. 22).

First we shall notice that FDs 4, 5, 6, 7, 10, 11 gives zero contribution $\left(\delta_{4}=\delta_{5}=\delta_{6}=\right.$ $\left.\delta_{7}=\delta_{10}=\delta_{11}=0\right)$ due to

$$
\int \frac{d^{4} k}{i \pi^{2}} \frac{\left(\mu k q\left(2 q_{-}-k\right)\right)}{k^{2}\left(k^{2}-2 k q_{-}\right)\left(k^{2}-2 k q+q^{2}-M^{2}\right)} \equiv 0 .
$$

FDs 2 and 3 are the contributions from pion wave function renormalization and they are equal to [5]:

$$
\delta_{c}=\delta_{2}+\delta_{3}=\frac{\alpha}{\pi}\left(L_{\Lambda}+\ln \frac{M^{2}}{\lambda^{2}}-\frac{3}{4}\right),
$$

where $L_{\Lambda}=\ln \left(\Lambda^{2} / M^{2}\right)$ and $\Lambda$ - is the ultraviolet cut-off parameter, $\lambda$ - is the fictitious photon mass. Considering contributions of FDs 1, 8, 9 we get:

$$
\begin{gathered}
\delta_{v}=\delta_{1}+\delta_{8}+\delta_{9}=\frac{\alpha}{\pi}\left[1+\frac{1}{2} L_{\Lambda}-\frac{1+\beta^{2}}{2 \beta} \ln \left(\frac{1+\beta}{1-\beta}\right) \ln \frac{M^{2}}{\lambda^{2}}+\right. \\
\left.+\frac{1+\beta^{2}}{4} s_{1} \operatorname{Re} \int_{0}^{1} \frac{d x}{q_{x}^{2}}\left(\ln \frac{q_{x}^{2}}{M^{2}}-2\right)+Q\right], \\
Q=\int_{0}^{1} d x \int_{0}^{1} d y y \ln \frac{-x(1-x y)+x x_{+}(1-y)+\mu^{2} y}{-x(1-x y)+x x_{-}(1-y)+\mu^{2} y} .
\end{gathered}
$$

Here $s_{1}=\left(q_{+}+q_{-}\right)^{2}$ is the invariant mass of charged pions pair, $\beta^{2}=1-4 M^{2} / s_{1}, q_{x}^{2}=$ $M^{2}-s_{1} x(1-x)-i 0$. The integrals in (9) can be calculated explicitly:

$$
\begin{aligned}
\operatorname{Re} \int_{0}^{1} \frac{d x s_{1}}{q_{x}^{2}} & =-\frac{2}{\beta} L, \\
\operatorname{Re} \int_{0}^{1} \frac{d x s_{2}}{q_{x}^{2}} \ln \frac{q_{x}^{2}}{M^{2}} & =\frac{4}{\beta}\left[L \ln \left(\frac{1+\beta}{2 \beta}\right)-\frac{1}{4} L^{2}+\mathrm{Li}_{2}\left(\frac{1-\beta}{1+\beta}\right)+2 \xi_{2}\right],
\end{aligned}
$$

where $L=\ln \frac{1+\beta}{1-\beta}, \operatorname{Li}_{2}(x)=-\int_{0}^{x} \frac{d t}{t} \ln (1-t)$ and $\xi_{2}=\frac{\pi^{2}}{6}$. 


\section{SOFT REAL PHOTON EMISSION}

The standard calculation of contribution of real soft photon emission by final pions

$$
\delta_{s}=\frac{\sigma_{\text {soft }}}{\sigma_{B}}=-\left.\frac{\alpha}{4 \pi^{2}} \int \frac{d^{3} k}{\omega}\left(\frac{q_{-}}{k q_{-}}-\frac{q_{+}}{k q_{+}}\right)^{2}\right|_{\omega<\Delta \varepsilon}
$$

where $\Delta \varepsilon$ is the maximum energy of soft photon (i.e. $\omega<\Delta \varepsilon$ ), leads to:

$$
\begin{aligned}
\delta_{s}=\frac{2 \alpha}{\pi}\{ & \left(\ln \Delta-\frac{1}{2} \ln \left(x_{+} x_{-}\right)+\ln \frac{M}{\lambda}\right)\left(-1+\frac{1+\beta^{2}}{2 \beta} L\right)+ \\
& \left.+\frac{1+\beta^{2}}{4 \beta}\left[-g-\frac{1}{2} L^{2}+L \ln \left(\frac{4}{1-\beta^{2}}\right)-\xi_{2}-2 \operatorname{Li}_{2}\left(-\frac{1-\beta}{1+\beta}\right)\right]\right\},
\end{aligned}
$$

where $\Delta=\Delta \varepsilon / \sqrt{s}$ and the quantity $g$ is defined by

$$
g=2 \beta \int_{0}^{1} \frac{d t}{1-\beta^{2} t^{2}} \ln \left(1+\frac{1-t^{2}}{4} \frac{\left(x_{+}-x_{-}\right)^{2}}{x_{+} x_{-}}\right) .
$$

\section{HARD REAL PHOTON EMISSION}

Consider the contribution of radiative corrections which arises from the emission of additional hard photon by final particles, i.e. the process:

$$
e^{-}\left(p_{-}\right)+e^{+}\left(p_{+}\right) \rightarrow \pi^{-}\left(q_{-}\right)+\pi^{+}\left(q_{+}\right)+\pi^{0}\left(q_{0}\right)+\gamma(k) .
$$

Amplitude for this process can be written in the form:

$$
M=(4 \pi \alpha)^{2} \frac{1}{q^{2}} \cdot \bar{v}\left(p_{+}\right) \gamma_{\mu} u\left(p_{-}\right) \cdot \frac{1}{4 \pi^{2} f_{\pi}^{3}} \cdot T_{\mu \nu} e^{\nu}(k),
$$

where $k, e_{\mu}(k)$ are the momenta and the polarization vector of final real photon. $T_{\mu \nu}$ is the tensor corresponding to the $\gamma^{*}(\mu, Q) \rightarrow \pi^{+}\left(q_{+}\right) \pi^{-}\left(q_{-}\right) \pi^{0}\left(q_{0}\right) \gamma(\nu, k)$ vertex, which follows from (11):

$$
\begin{aligned}
T^{\mu \nu}= & (\mu \nu Q k) A+\left(\mu \nu(Q+k) q_{0}\right)+\left(\mu \lambda Q q_{0}\right)\left(\frac{q_{-}^{\nu} q_{+}^{\lambda}}{\left(q_{-} k\right)}+\frac{q_{+}^{\nu} q_{-}^{\lambda}}{\left(q_{+} k\right)}\right)- \\
& -\left(\nu \lambda k q_{0}\right)\left(\frac{\left(2 q_{-}-Q\right)^{\mu} q_{+}^{\lambda}}{Q^{2}-2\left(q_{-} Q\right)}+\frac{\left(2 q_{+}-Q\right)^{\mu} q_{-}^{\lambda}}{Q^{2}-2\left(q_{+} Q\right)}\right)
\end{aligned}
$$

where $Q=q_{+}+q_{-}+q_{0}+k$, (thus $\left.Q_{0}=\sqrt{s}\right), A=1-\left(s_{1}-M^{2}\right) /\left((Q-k)^{2}-M^{2}\right)$. This tensor satisfies the gauge-invariance condition for both photon legs:

$$
Q_{\mu} T^{\mu \nu}=k_{\nu} T^{\mu \nu}=0
$$


Thus we may write the cross section in a form:

$$
\sigma^{\text {hard }}=\frac{(4 \pi \alpha)^{3}}{8 s} \cdot \frac{1}{s^{2}} S p\left[\hat{p}_{+} \gamma^{\mu} \hat{p}_{-} \gamma^{\nu}\right]\left(\frac{1}{4 \pi^{2} f_{\pi}^{3}}\right)^{2} \frac{1}{3}\left(g_{\mu \nu}-\frac{Q_{\mu} Q_{\nu}}{Q^{2}}\right) \int d \Gamma_{4} \sum_{\lambda}\left|T^{\alpha \beta} e_{\beta}^{\lambda}\right|^{2} .
$$

Phase volume for final state has a form:

$$
\begin{aligned}
d \Gamma_{4} & =(2 \pi)^{4} \delta^{4}\left(p_{+}+p_{-}-q_{+}-q_{-}-q_{0}-k\right) \frac{d^{3} q_{+}}{(2 \pi)^{3} 2 \varepsilon_{+}} \frac{d^{3} q_{-}}{(2 \pi)^{3} 2 \varepsilon_{-}} \frac{d^{3} q_{0}}{(2 \pi)^{3} 2 \varepsilon_{0}} \frac{d^{3} k}{(2 \pi)^{3} 2 \omega}= \\
& =(2 \pi)^{-8} \frac{s^{2} \pi^{2}}{16} x d x d x_{+} d x_{-} d O_{\gamma},
\end{aligned}
$$

where $x=\omega / \sqrt{s}, x+x_{+}+x_{-}+x_{0}=1$. And now the cross section takes the form:

$$
\sigma^{\text {hard }}=\left.\frac{\alpha^{3}}{2^{8} \cdot 3 \cdot \pi^{7} f_{\pi}^{6}} \int d x x d x_{+} d x_{-} d O_{\gamma}\left(-\sum_{\lambda}\left|T^{\mu \nu}\right|^{2}\right)\right|_{x>\Delta} .
$$

We shall notice, that the sum of hard photon emission $\delta_{h}=\sigma^{\text {hard }} / \sigma_{B}$ and soft real photon emission $\delta_{s}$ contributions $\left(\delta_{h}+\delta_{s}\right)$ does not contain the auxiliary parameter $\Delta$. To see this explicitly let us consider the small $x=\omega / \sqrt{s}$ limit of $\sigma^{\text {hard }}$. Really if we consider the case $\Delta \sqrt{s}<\omega \ll \sqrt{s}$ then (see (18)):

$$
\left.T^{\mu \nu} e_{\nu}(k)\right|_{\omega \ll \sqrt{s}} \approx\left(\mu q_{+} q_{-} q_{0}\right)\left(\frac{\left(q_{-} e\right)}{\left(q_{-} k\right)}-\frac{\left(q_{+} e\right)}{\left(q_{+} k\right)}\right) .
$$

We can calculate the hard photon emission contribution in this limit:

$$
\left.\int \frac{d^{3} k}{2 \pi \omega} \sum_{\lambda}\left(-\left|T^{\mu \nu} e_{\nu}(k)\right|^{2}\right)\right|_{\omega \rightarrow \Delta \sqrt{s}}=4 \ln \frac{1}{\Delta}\left(\frac{1+\beta^{2}}{2 \beta} \ln \left(\frac{1+\beta}{1-\beta}\right)-1\right),
$$

and we get for $\delta_{h}$ :

$$
\left.\delta_{h}\right|_{\omega \rightarrow \Delta \sqrt{s}} \approx \frac{2 \alpha}{\pi}\left[\ln \frac{1}{\Delta}\left(\frac{1+\beta^{2}}{2 \beta} \ln \left(\frac{1+\beta}{1-\beta}\right)-1\right)+O(\Delta)\right] .
$$

We redefine the contributions in the following manner:

$$
\begin{gathered}
\delta_{s}+\delta_{h} \rightarrow \bar{\delta}_{s}+\bar{\delta}_{h}, \\
\bar{\delta}_{s}=\delta_{s}+\frac{2 \alpha}{\pi} \ln \frac{1}{\Delta}\left(\frac{1+\beta^{2}}{2 \beta} \ln \left(\frac{1+\beta}{1-\beta}\right)-1\right), \\
\bar{\delta}_{h}=\delta_{h}-\frac{2 \alpha}{\pi} \ln \frac{1}{\Delta}\left(\frac{1+\beta^{2}}{2 \beta} \ln \left(\frac{1+\beta}{1-\beta}\right)-1\right),
\end{gathered}
$$

where both $\bar{\delta}_{s}$ and $\bar{\delta}_{h}$ are not dependent on $\Delta$ any more. 


\section{CONCLUSION}

The final result is

$$
\begin{aligned}
\sigma^{e e \rightarrow 3 \pi(\gamma)} & =\sigma_{B}^{(0)}\left(1+\delta_{s w}\right)(1+\delta)=\sigma_{B}(1+\delta), \quad \sigma_{B}=\sigma_{B}^{(0)}\left(f_{\pi}^{(0)} \rightarrow f_{\pi}\right) \\
\delta & =\left.\left(\delta_{c}+\delta_{v}+\bar{\delta}_{s}+\bar{\delta}_{h}\right)\right|_{L_{\Lambda}=0}, \quad \delta_{s w}=\frac{3 \alpha}{2 \pi} L_{\Lambda}
\end{aligned}
$$

where we extracted the short-distance contributions in form $\left(1+\delta_{s w}\right)$ and used this factor to renormalize $f_{\pi}^{(0)}$ pion decay constant in form $f_{\pi}^{(0)-6}\left(1+\delta_{s w}\right)=f_{\pi}^{-6}[\underline{6}]$.

The explicit form of $\delta-\bar{\delta}_{h}$ is:

$$
\begin{aligned}
\delta-\bar{\delta}_{h}=\frac{\alpha}{\pi}\{ & -\frac{1}{2} \ln \left(x_{+} x_{-}\right)\left(-1+\frac{1+\beta^{2}}{2 \beta} L\right)+\frac{1}{4}+Q+ \\
& +\frac{1+\beta^{2}}{4 \beta}\left[-g-\frac{1}{2} L^{2}+L \ln \frac{4}{1-\beta^{2}}-\xi_{2}-2 \operatorname{Li}_{2}\left(-\frac{1-\beta}{1+\beta}\right)\right]+ \\
& \left.+\frac{1+\beta^{2}}{\beta}\left[L+L \ln \frac{1+\beta}{2 \beta}-\frac{1}{4} L^{2}+\operatorname{Li}_{2}\left(-\frac{1-\beta}{1+\beta}\right)+2 \xi_{2}\right]\right\},
\end{aligned}
$$

with $L=\ln \left(\frac{1+\beta}{1-\beta}\right), g$ defined in (15) and $Q$ defined in (10). We note that $g\left(x_{+}=x_{-}\right)=$ $Q\left(x_{+}=x_{-}\right)=0$. The form of $\bar{\delta}_{h}$ depends on the experimental conditions of final state particles registration and not considered here.

In Fig. 3 we present the value $\delta-\bar{\delta}_{h}$ for typical experimental situation.

\section{Acknowledgments}

We are grateful to G. V. Fedotovich for attracting our attention to this problem. We are also grateful to Z. K. Silagadze for valuable discussions.

[1] G. W. Bennett et al. [Muon g-2 Collaboration], Phys. Rev. Lett. 92, 161802 (2004) arXiv:hep-ex/0401008.

[2] Y. M. Bystritskiy, E. A. Kuraev, G. V. Fedotovich and F. V. Ignatov, arXiv:hep-ph/0505236.

[3] E. Witten, Nucl. Phys. B 223, 433 (1983).

[4] J. Wess and B. Zumino, Phys. Lett. B 37 (1971) 95.

[5] Ngee-Pong Chang, Phys. Rev. 131 (1963) 1272.

[6] B. R. Holstein, Phys. Lett. B 244, 83 (1990). 


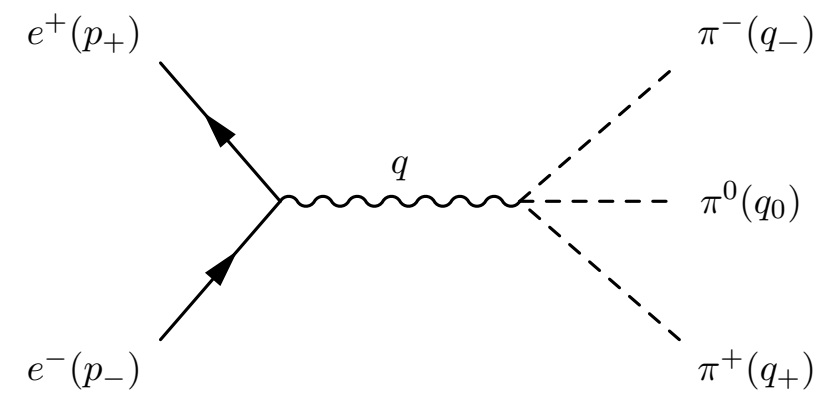

Fig. 1: Feynman diagram contributing the process probability in Born approximation.

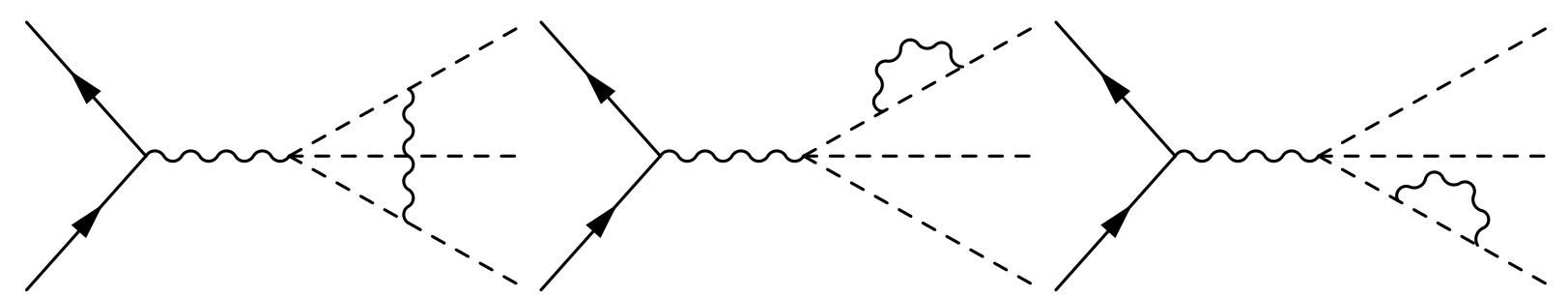

1

2

3

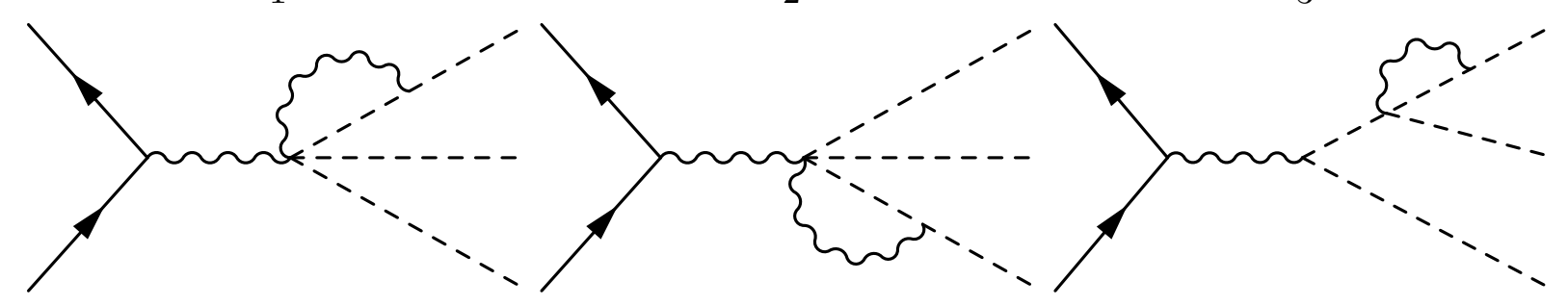

4

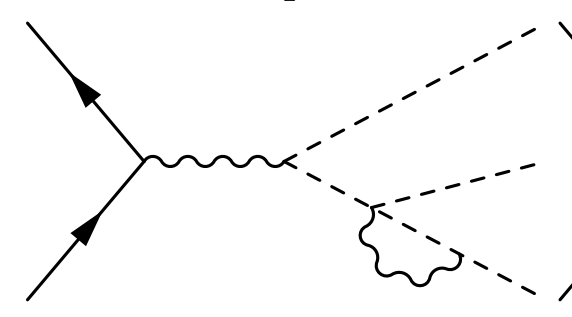

5

6

7
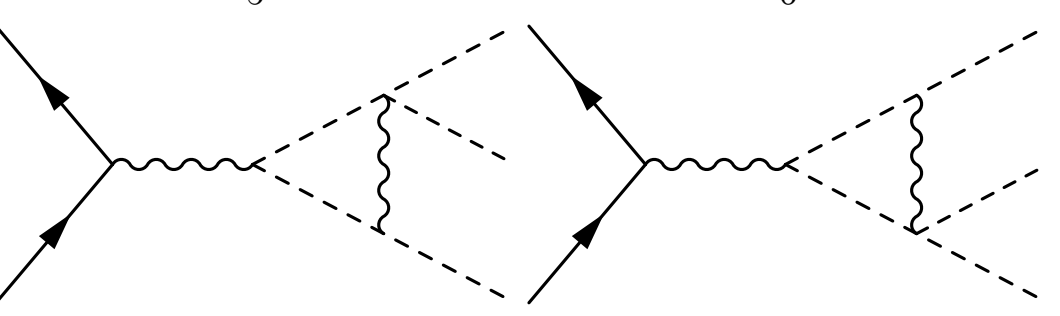

8

9

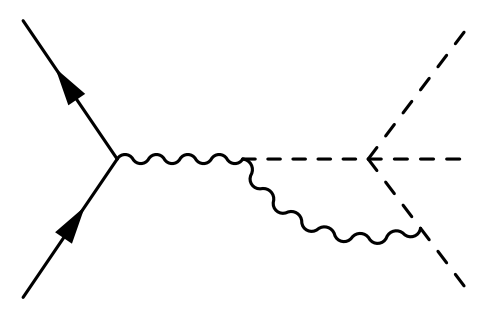

10

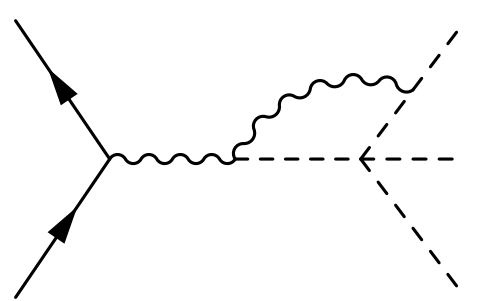

11

Fig. 2: Feynman diagrams of emission of additional virtual photon. 


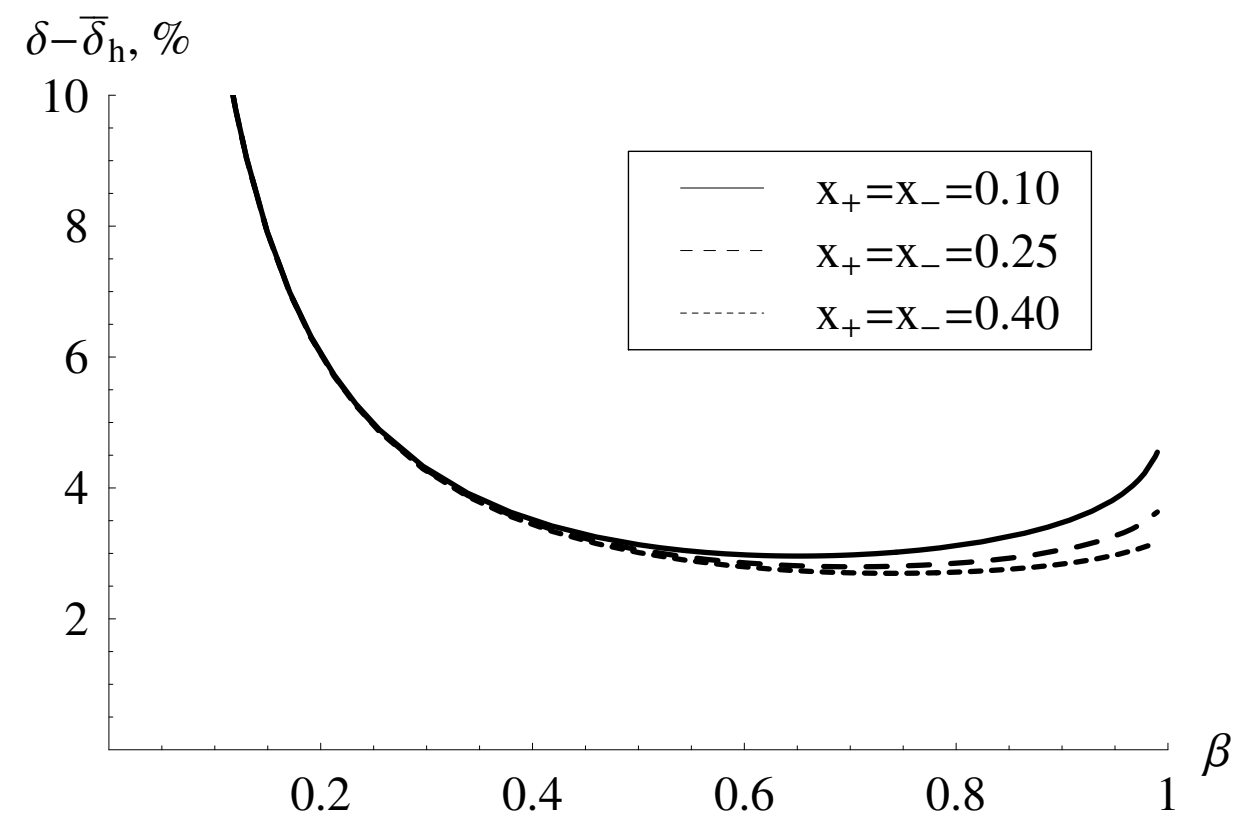

Fig. 3: The value $\delta-\bar{\delta}_{h}$ (see (31) ) in percents for typical experimental conditions as function of $\beta$. 